\title{
Supplementary materials: Optical focusing beyond the diffraction limit via vortex-assisted transient microlenses
}

\author{
Eitan Edrei And Giuliano Scarcelli*
}

Fischell Department of Bioengineering, University of Maryland, College Park, Maryland 20742, USA

*Corresponding author: scarc@umd.edu

\section{Theory}

Following the formalism and derivation of Gordon et al, ${ }^{1}$ we consider the geometrical configuration shown in figure $\mathrm{S} 1$. A collimated beam of radius $\omega_{0}$ is transmitted through a slab of thickness $l$ with a radially decreasing refractive index centered at the optical axis. We seek an expression for the distance $F$ from the slab in which the focal point is obtained.

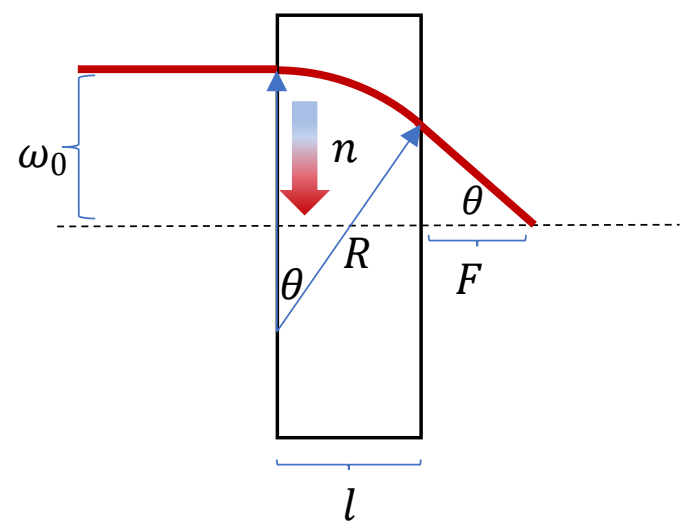

Figure S1: Schematic of the focusing geometry

A beam propagating through the varying refractive index profile will follow the trajectory given by: ${ }^{2}$

$$
\frac{1}{R}=\frac{1}{n}\left(\frac{d n}{d r}\right)
$$

Where $r$ is the radial coordinate with respect to the optical axis. Assuming a parabolic refractive index variation of the form:

$$
n=n_{0}\left[1+\delta\left(\frac{r}{\omega_{0}}\right)^{2}\right]
$$

Substituting S2 in equation S1 the radius of curvature can be obtained:

$$
R \approx \frac{\omega_{0}^{2}}{2 \delta r}
$$

Assuming $l \ll F$ (not drawn to scale), from geometrical considerations we can approximate the expression for the focal distance: 


$$
F \sim \frac{r}{\theta} \sim \frac{r R}{l}(\mathrm{~S} 4)
$$

Substituting S3 in S4 the focal distance takes the form:

$$
F \approx \frac{\omega_{0}^{2}}{l \cdot 2 \delta}
$$

To estimate the value of $\delta$ we need to obtain the heat profile from which the refractive index can be evaluated using:

$$
n(r, t)=n_{0}+\left(\frac{d n}{d T}\right) \Delta T(r, t)
$$

Solving the heat diffusion equation for a singular heat source yields: ${ }^{1}$

$$
\Delta T(r, t) \approx \frac{0.06 b \cdot P}{\pi k} \cdot\left[\ln \left(1+\frac{8 D t}{\omega_{0}^{2}}\right)-\frac{16 D t}{\omega_{0}^{2}+8 D t} \cdot \frac{r^{2}}{\omega_{0}^{2}}\right]
$$

Where D is the heat diffusion coefficient. By substituting the $r$ dependent term of S7 into S6 and comparing it to the expression of S2 the value of $\delta$ can be extracted:

$$
\delta=\left(\frac{d n}{d T}\right) \frac{0.06 b \cdot P}{\pi k n_{0}} \cdot \frac{16 D t}{\omega_{0}^{2}+8 D t}
$$

Substituting S8 into S5 we obtain the time-dependent expression for the focal length:

$$
F(t)=\frac{\pi n_{0} k \omega_{0}^{2}\left(\omega_{0}^{2}+8 D t\right)}{0.24 b P l(d n / d T) \cdot 8 D t}(\mathrm{~S} 9)
$$

If we define a constant time scale as: $t_{c}=\frac{\omega_{0}^{2}}{4 D}$ and the focal length at steady-state as: $F_{\infty}=$ $\frac{k \pi n_{0} \omega_{0}^{2}}{0.24 b P l(d n / d T)}$ the expression in $\mathrm{S} 9$ can be rearranged to the form:

$$
F(t)=F_{\infty} \cdot\left[1+\frac{t_{c}}{2 t}\right]
$$

Where the $t^{-1}$ behavior is clearly seen.

So far, we have discussed a scenario in which heat diffusion is significant and needs to be accounted for. If high intense laser power $(\sim 1-10 \mathrm{~mJ})$ is available within a short pulse, the diffusion effect can be neglect; the temperature and hence the refractive index profile will closely follow the light pattern which can be designed at will. Let us assume that in the ring profile the temperature reaches $359 \mathrm{~K}$ (see main text regarding damage thresholds), while the central portion remains at room temperature, i.e. $293 \mathrm{~K}$. This can be achieved only in the absence of diffusion, i.e. thermal confinement. The refractive index difference in such case is $\sim 0.009 .{ }^{3}$ From equation S2 we can estimate the value of $\delta$ to be 0.0068 , assuming an absorbing length of $\sim 100 \mu \mathrm{m}$ the focal length $\mathrm{F}$ can be evaluated from equation S5 and the focal size from equation 3 of the main text. We approximate the focal size to be $\sim 0.35 \omega_{0}$, close to the value achieved in figure $2 \mathrm{e}$

\section{Dye properties}


In this work we designed an absorbing sample to increase the absorption efficiency of our laser in the water-based solutions. However, with an appropriate match between the sample and the laser wavelength this step can be removed in future studies. The absorption spectra of the dye is shown in figure S2:

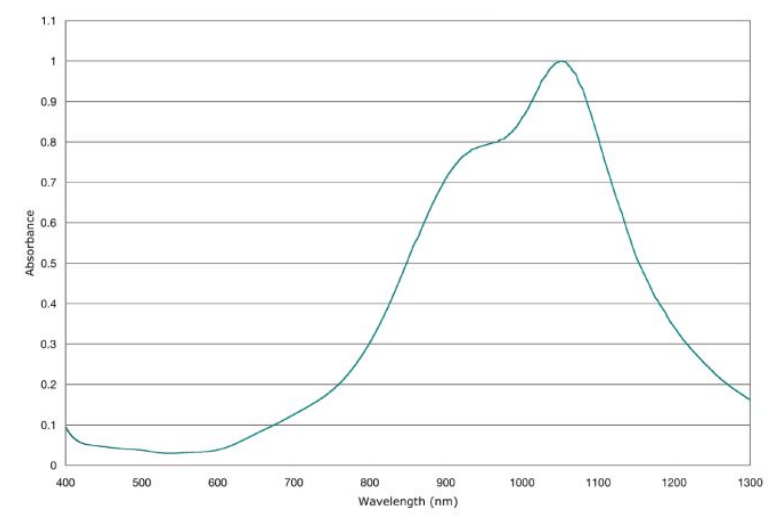

Figure S2: Dye absorption spectra

\section{Simulation}

To simulate the expected heat profile, we used COMSOL Multiphysics software. The geometry of the model and the heat profile results after $5 \mathrm{~ms}$ of absorption are shown in figure S3 (spatial axes are presented in unites of micrometers and the temperature scalebar is in Kelvin). The exterior boundaries of the structure were kept at room temperature $(T=293.15 \mathrm{~K})$, material properties such as thermal conductivity and density were taken from the built-in parameters of the software (the solution was assumed to have similar properties as water). The ring profile thickness was set to 30 $\mu \mathrm{m}$ since the absorptivity of the material is $\sim 30 \frac{\mathrm{L}}{\mathrm{g} \cdot \mathrm{cm}}$, thus, after $30 \mu \mathrm{m}$ the intensity drops to $e^{-1}$ of the initial intensity. The total power absorbed by the ring structure was set to $0.075 \mathrm{~W}$, in agreement with the value of $0.1 \mathrm{~W}$ used in our experiment and considering the relative absorption in the $30 \mu \mathrm{m}$ scale. From the heat distribution the refractive index map can be calculated using pre-measured values of water refractive index at different temperatures. ${ }^{3}$ 


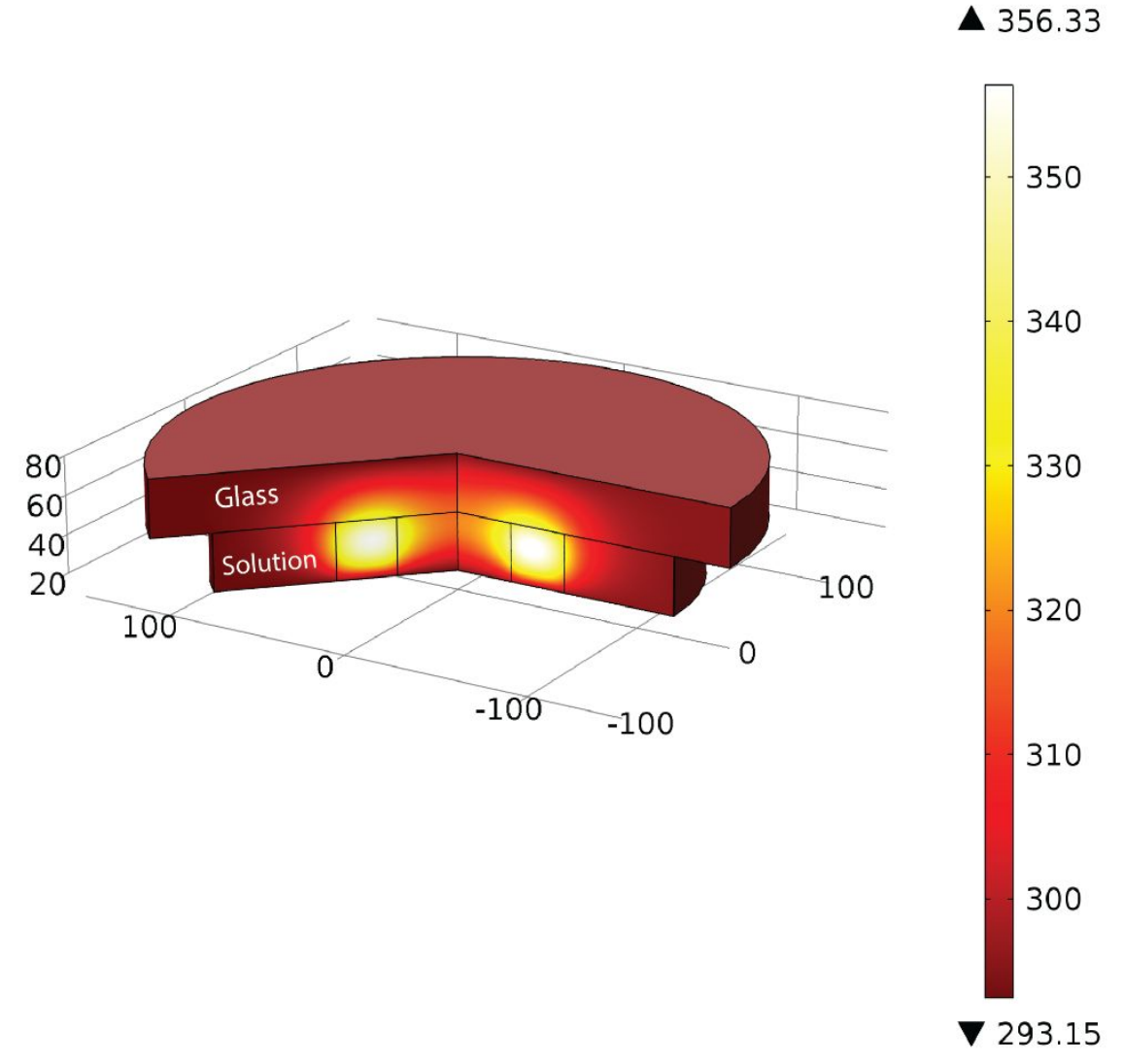

Figure S3: Heat diffusion model: A heat source was defined in a ring-shaped configuration; exterior boundaries were set to room temperature. The heat profile was modeled for various times.

(1) Gordon, J.; Leite, R.; Moore, R. S.; Porto, S.; Whinnery, J. Long-transient effects in lasers with inserted liquid samples. Journal of Applied Physics 1965, 36 (1), 3-8.

(2) Born, M.; Wolf, E. Principles of optics: electromagnetic theory of propagation, interference and diffraction of light, Elsevier: 2013.

(3) Wei, T.; Han, Y.; Li, Y.; Tsai, H.-L.; Xiao, H. Temperature-insensitive miniaturized fiber inline FabryPerot interferometer for highly sensitive refractive index measurement. Optics Express 2008, 16 (8), 5764-5769. 